\title{
INFLUENCE OF PHASE CHANGE ON SELF-PRESSURIZATION IN CRYOGENIC TANKS UNDER MICROGRAVITY
}

\author{
Juan $\mathrm{Fu}^{1,2}$, Bengt Sunden ${ }^{2 *}$, Xiaoqian Chen ${ }^{1}$, Yiyong Huang ${ }^{1}$ \\ [1] College of Aerospace Science and Engineering, National University of Defense Technology, Changsha \\ 410073, Hunan, P.R. China \\ [2] Department of Energy Sciences, Lund University, Lund 22100, Sweden \\ (*Corresponding author: bengt.sunden@energy.lth.se, Phone: +46 46222 8605, Fax: +46 46222 4717)
}

\begin{abstract}
Future operations of many fluid, thermal and power systems and their ability to store, transfer, and manage a variety of single or multiphase fluids in reduced gravity environment are of great importance. For many of these systems, cryogenic conditions will play an important role. Cryogenic vaporization, caused by heat leakage into the tank from the surrounding environment, is one of the main causes of mass loss and leads to self-pressurization of the storage tanks. Available publications on self-pressurization and stratification of cryogenic tanks mainly focus on the convection and surface evaporation influences. Because large superheats increase the likelihood of evaporation in the liquid, the evaporation and its effect on vapor pressure under microgravity is studied numerically in this paper. The effects of reduced gravity, contact angle of the vapor bubble, and surface tension are investigated. The computations are carried out by using the CFD software package, Ansys Fluent, and an in-house developed code to calculate the source term associated with the phase change. A coupled level set and the volume-of-fluid method (CLSVOF) are used to solve a single set of conservation equations for the whole domain and the interface between the two phases is tracked or captured. A heat and mass transfer model is implemented into the Fluent code for solving problems involving evaporation or condensation. Results show that small tiny vapor regions caused by the evaporation process change the pressure rise. Vortices are observed due to the vapor dynamics.
\end{abstract}

KEY WORDS: Self-pressurization, Evaporation, Reduced gravity, Cryogenics

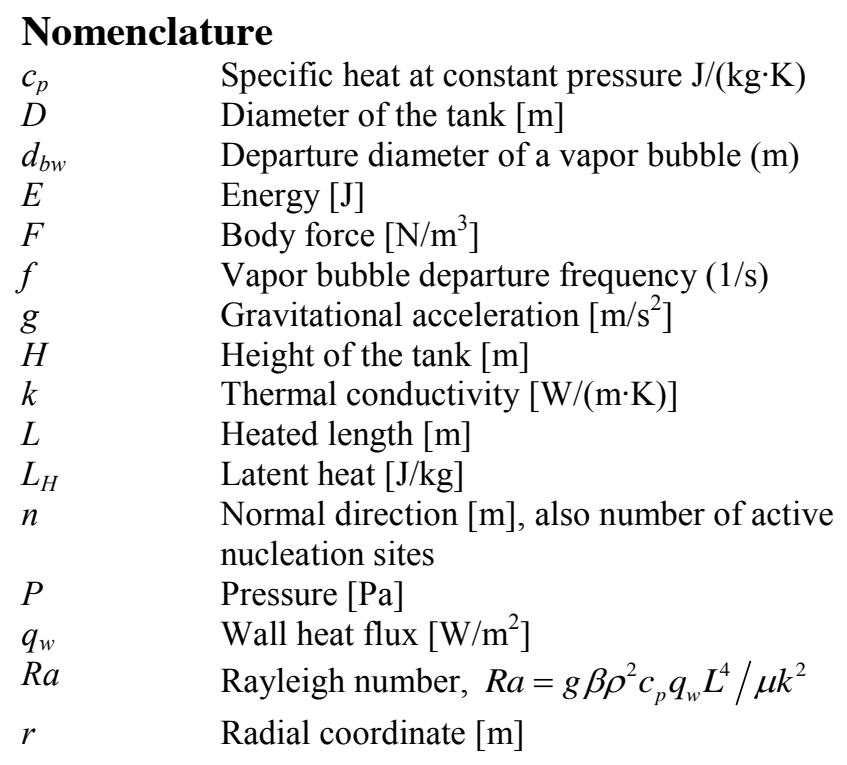




$\begin{array}{ll}S_{h} & \text { Source term in energy equation }\left[\mathrm{W} / \mathrm{m}^{3}\right] \\ s & \text { Width of the rib }[\mathrm{m}] \\ T & \text { Temperature }[\mathrm{K}] \\ t & \text { Time }[\mathrm{s}] \\ t_{g} & \text { Growth time }[\mathrm{s}] \\ t_{w} & \text { Waiting time }[\mathrm{s}] \\ \vec{V} & \text { Velocity vector }[\mathrm{m} / \mathrm{s}] \\ x, y, z & \text { Coordinates }[\mathrm{m}] \\ & \\ \text { Greek symbols } \\ \alpha & \text { Volume of fluid fraction }[-] \\ \alpha & \text { Heat transfer coefficient }\left[\mathrm{W} / \mathrm{m}^{2} \mathrm{~K}\right] \\ \beta & \text { Coefficient of thermal expansion }\left[\mathrm{K}^{-1}\right] \\ \varphi & \text { Level set function } \\ \Gamma & \text { Interface } \\ \kappa & \text { Curvature }[1 / \mathrm{m}] \\ \mu & \text { Dynamic viscosity }\left[\mathrm{m}^{2} / \mathrm{s}\right] \\ \rho & \left.\text { Density [kg/m }{ }^{3}\right] \\ \sigma & \text { Surface tension }[\mathrm{N} / \mathrm{m}] \\ \theta & \text { Contact angle } \\ \psi & \text { Stream function } \\ \text { Subscripts } & \\ c & \text { Convection } \\ c r & \text { Critical } \\ e & \text { Evaporation } \\ h & \text { Heat } \\ l & \text { Liquid } \\ q & \text { Quenching } \\ s a t & \text { Saturation } \\ v & \text { Superheat } \\ & \text { Vapor } \\ & \end{array}$

\section{INTRODUCTION}

Cryogenic liquids like liquid hydrogen and liquid oxygen play an integral role in aerospace missions due to their high efficient thrust and non-pollution waste. The cryogens are usually stored at very low temperature and the storage tanks are equipped with high quality insulation. However, heat leakage still occurs by conduction to the support structure and the pipelines that connect the tank to the other devices. The heat will be carried to the liquid-vapor interface by conduction and natural convection causing vaporization, which in a closed tank will result in a pressure rise. To control the self-pressurization and the pressure as well as the thermal stratification in cryogenic storage tanks is important for, e.g., space missions. Several experimental and numerical studies on the ground have been performed. Some of these were reviewed in two recent papers by Barsi and Kassemi [1,2]. In the first paper they focused on an experimental study while in the second one their concern was a numerical study. A meaningful prediction ability of the self-pressurization phenomenon in such cryogenic liquid tanks is critical and necessary for the pressure/thermal control and the design of the tank.

A number of investigators have developed approximate methods to obtain better agreement with experimental data. A homogeneous thermodynamic analysis was one of the earliest models developed to predict the self-pressurization rate in a cryogenic tank partially filled by a liquid. The agreement between the simple thermodynamic model and experiments has generally been poor especially in the initial transient regime with the assumption of the same energy increase rate for both the liquid and gas parts [3]. Transport effects in the fluid must be included. Hochstein et al. [4] developed an effective conductivity model for the 
liquid transport to account for evaporation without considering the gas-phase transport. Results were reasonable as compared with experiment [5] when the tank was heated uniformly from the bottom under normal gravity. However, deviations were noted for the top heating test case on the ground and for low gravity uniform heating. Amirkhanyan and Cherkasov [6] coupled one-dimensional conduction in the liquid to a lumped model of the vapor phase. The agreement between computational and experimental results was satisfactory for high liquid fill levels. However, the pressure rise was overpredicted at small fill levels. Barsi and Kassemi [7] employed a two-phase CFD model developed by Panzarella and Kassemi [8] that described the thermal stratification in the liquid to obtain the amount of evaporation at the interface with the assumption of a lumped vapor state. The model was compared with the pressurization test results at NASA Glenn's plum Brook Station [9]. It gave reasonable prediction for a low liquid fraction case, but a discrepancy was found for other liquid fraction cases. Roh and Son [10] neglected the vapor side transport contribution to the gas generation due to evaporation in a cryogenic liquid natural gas tank but no experimental comparisons were attempted. Grayson et al. [11] included transport in the vapor and developed a model for the phase change effects at the liquid-gas interface in the so-called FLOW-3D code. Experimental validation for the CFD model was achieved for cryogenic tanks in low-gravity with a high heat leak and big vapor volume. Most of these numerical studies mainly focused on the convection and surface evaporation influences. When the superheat of the liquid close to the tank wall reaches a certain value, evaporation occurs. The appearance of vapor regions changes the heat transfer and fluid flow.

In this study, a further development of our previous research works [12, 13] is carried out. In particular, evaporation and its effect on vapor pressure under microgravity are studied in a closed partially filled liquid hydrogen tank by employing a coupled level set and volume-of-fluid method (CLSVOF) [14] and the phase change effect is also considered. The phenomenon is investigated in a partially filled liquid hydrogen tank for different values of the gravity acceleration and the effects of contact angle and surface tension are revealed.

\section{MATHEMATICAL MODEL}

An axi-symmetric cylindrical tank partially filled with liquid hydrogen is considered, as shown in Fig. 1. Only hydrogen vapor is considered to be present in the vapor space. The VOF method [15], which is a kind of an Eulerian method, is adopted here. Previously it has been extensively used in predicting two-phase fluid flows, see [16-18]. The VOF formulation relies on the fact that two or more fluids are not interpenetrating each other. For each phase considered in the model, a variable is introduced as the volume fraction of the phase in the computational cell. In each of the control volumes, the volume fractions of all phases sum up to unity. Because the temperature changes only slightly, all of the fluid properties, except the density, are considered as constants.

The density variation attributed to temperature is described by the Boussinesq approximation for the evaluation of the buoyancy force. In order to simplify the solution procedure, the governing equations for conservation of mass, momentum, and energy are

$$
\begin{gathered}
\frac{\partial \rho}{\partial t}+\nabla \cdot(\rho \vec{V})=0 \\
\frac{\partial(\rho \vec{V})}{\partial t}+\nabla \cdot(\rho \vec{V} \vec{V})=-\nabla p-\rho \beta \vec{g}\left(T-T_{0}\right)+\nabla \cdot\left[\mu_{e f f}\left(\nabla \vec{V}+\nabla \vec{V}^{T}\right)\right]+\vec{F} \\
\frac{\partial(\rho E)}{\partial t}+\nabla \cdot(\vec{V}(\rho E+p))=\nabla \cdot\left(k_{e f f} \nabla T\right)+S_{h}
\end{gathered}
$$

where $\vec{F}$ is the body force resulting from surface tension at the interface and $S_{h}$ is an energy source of heat exchange related to the phase change process. 
By using the so-called continuum surface force (CSF) model, the surface tension force is converted into a volume force as developed by Brackbill et al. [19]. The volume force acts as a source term in the momentum equation and has the following form:

$$
F_{v o l}=\sigma_{l v} \frac{\alpha_{l} \rho_{l} \kappa_{v} \nabla \alpha_{v}+\alpha_{v} \rho_{v} \kappa_{l} \nabla \alpha_{l}}{0.5\left(\rho_{l}+\rho_{v}\right)}
$$

where $\sigma_{l v}$ is the interfacial tension between the liquid and vapor. The curvatures of liquid and vapor are defined as:

$$
\kappa_{l}=\frac{\nabla \alpha_{l}}{\left|\nabla \alpha_{l}\right|}, \quad \kappa_{v}=\frac{\nabla \alpha_{v}}{\left|\nabla \alpha_{v}\right|}
$$

The continuum surface force model was originally introduced and derived by Brackbill et al. [19]. It has then been extensively and successfully used by many investigators, e.g., [20-22], and in particular for phase change problems by [23-25].

The tracking of the interface between the phases is accomplished by the solution of a continuity equation for the volume fraction of the second phase.

$$
\frac{\partial}{\partial t}\left(\alpha_{v} \rho_{v}\right)+\nabla \cdot\left(\alpha_{v} \rho_{v} \vec{V}\right)=\dot{m}
$$

Here $\dot{m}$ means the phase change due to evaporation or condensation at the interface, positive and negative, respectively. It is regarded as a source term in the continuity equation. The Lee phase change model [26] is applied to consider this mass transfer and it is described by:

$$
\begin{aligned}
& \dot{m}=r_{l} \alpha_{l} \rho_{l}\left(T_{l}-T_{\text {sat }}\right) / T_{\text {sat }} \quad T_{l} \geq T_{\text {sat }} \\
& =r_{v} \alpha_{v} \rho_{v}\left(T_{v}-T_{\text {sat }}\right) / T_{\text {sat }} \quad T_{v}<T_{\text {sat }}
\end{aligned}
$$

The saturation temperature $T_{\text {sat }}$ changes according to the pressure in the tank by the Clausius-Clapeyron equation. The coefficient $r$ is found by a trial-and-error procedure as described in [27]. Thus the energy source term $S_{h}$ will be:

$$
S_{h}=L_{H} \dot{m}
$$

where $L_{H}$ is the latent heat of liquid hydrogen.

The properties appearing in the transport equations are determined by the presence of the component phases in each control volume. For instance, thermal conductivity, density, dynamic viscosity, and specific heat are commonly computed by the following expressions:

$$
\begin{aligned}
& k=\alpha_{l} k_{l}+\left(1-\alpha_{l}\right) k_{v} \\
& \rho=\alpha_{l} \rho_{l}+\left(1-\alpha_{l}\right) \rho_{v} \\
& \mu=\alpha_{l} \mu_{l}+\left(1-\alpha_{l}\right) \mu_{v} \\
& c_{p}=\frac{1}{\rho}\left[\alpha_{l} \rho_{l} c_{p l}+\left(1-\alpha_{l}\right) \rho_{v} c_{p v}\right]
\end{aligned}
$$


The level-set method is a popular interface-tracking method for computing two-phase flows with topologically complex interfaces. This is similar to the interface tracking method of the VOF model. In the level-set method [28], the interface is captured and tracked by the level-set function, defined as an assigned distance from the interface.

The level-set function $\varphi$ is defined as an assigned distance to the interface. Accordingly, the interface is the zero level-set $\varphi(x, t)$ and can be expressed as $\Gamma=\{x \mid \varphi(x, t)=0\}$ in a two-phase flow system:

$$
\varphi(x, t)= \begin{cases}+|\mathrm{d}| & \text { if } x \in \text { the primary phase } \\ 0 & \text { if } x \in \Gamma \\ -|\mathrm{d}| & \text { if } x \in \text { the secondary phase }\end{cases}
$$

where $d$ is the distance from the interface. The normal and curvature of the interface, which are needed in the computation of the surface tension force, can be estimated as:

$$
\vec{n}=\left.\frac{\nabla \varphi}{|\nabla \varphi|}\right|_{\varphi=0}, \kappa=\left.\nabla \cdot \frac{\nabla \varphi}{|\nabla \varphi|}\right|_{\varphi=0}
$$

The evolution of the level-set function is given in a similar fashion as for the VOF model:

$$
\frac{\partial \varphi}{\partial t}+\nabla \cdot(\vec{V} \varphi)=0
$$

The momentum equation can be written as:

$$
\begin{aligned}
\frac{\partial(\rho \vec{V})}{\partial t}+\nabla \cdot(\rho \vec{V} \vec{V})= & -\nabla p-\rho \beta \vec{g}\left(T-T_{0}\right)+\nabla \cdot\left[\mu_{e f f}\left(\nabla \vec{V}+\nabla \vec{V}^{T}\right)\right] \\
& -\sigma \kappa \delta(\varphi) \nabla \varphi
\end{aligned}
$$

In. Eq. (13), $\delta(\varphi)=(1+\cos (\pi \varphi / a)) / 2 a$ if $|\varphi|<a$ and $a=1.5 h$ (where $h$ is the grid spacing), otherwise $\delta(\varphi)=0 . \sigma$ is the surface tension.

The boundary conditions for the studied problem (see Fig. 1) are formulated as follows. The top and bottom surfaces of the tank are assumed to be flat and perfectly insulated. Heat in-leak takes place at the cylindrical wet-walls and different rates of the heat in-leak $q_{w}$ might be specified. The heating of liquid hydrogen at the walls induces free convection currents with the warmer liquid hydrogen in the wall region being forced to the upper regions of the liquid column.

Quiescent saturation conditions are assumed before imposing the heat flux $q_{w}$ at the cylindrical walls. The initial conditions at $\mathrm{t}=0$ are:

$$
u(r, z)=u_{r}=u_{z}=0
$$

The initial pressure is set to one atmosphere and the initial temperature corresponds to the saturation temperature at that pressure. The initial temperature is assumed constant throughout the liquid and vapor. 
The pressure in the liquid is taken as a function of the height and density. No slip boundary conditions are imposed on the sidewalls.

At the top and bottom surfaces, an adiabatic boundary condition is assumed which gives:

$$
\frac{\partial T}{\partial n}=0
$$

whereas on the side walls the Neumann boundary condition is applied, i.e.:

$$
-k \frac{\partial T}{\partial n}=q_{w}
$$

\section{NUMERICAL IMPLEMENTATION}

The commercial CFD code Fluent is used to solve the conservation equations. The interfacial mass and heat transfer model is implemented via user-defined functions. Because the geometry, boundary conditions, and physics of the problem demand symmetry conditions, the flow is treated as axisymmetric and the Rayleigh number $R a^{*}=g \beta \rho^{2} c_{p} q_{w} L^{4} / \mu \lambda^{2}$ is used to determine the flow type. The tank has a diameter of $0.5 \mathrm{~m}$ and a height of $1 \mathrm{~m}$. A heat flux of $250 \mathrm{~W} / \mathrm{m}^{2}$, which corresponds to the overall heat in-leak in the large insulated liquid hydrogen tank [29], is imposed to the tank with a fill level of 50\% at microgravity and zero gravity. For the self-pressurization problem considered, the Rayleigh number characterizing the flow type in the liquid is $1.05 \times 10^{12}$ for a gravity acceleration of $10^{-3} \mathrm{~g}_{0}$. The magnitude of the Rayleigh number indicates that the natural convection in the liquid is turbulent [30]. In zero gravity condition, laminar flow prevails and the Rayleigh number is zero, i.e., no buoyancy force acts and the surface tension becomes more important. Contact angles of 5 degree and 10 degree are employed to study the effect of the contact angle on the pressure rise and fluid flow. The effect of different surface tension is studied by considering the values $\sigma=0.002 \mathrm{~N} / \mathrm{m}$ and $\sigma=0.005 \mathrm{~N} / \mathrm{m}$, respectively.

The SIMPLEC (Semi-Implicit Method for Pressure Linked Equation-Consistent) is chosen as the pressurevelocity coupling algorithm, which has an improved behavior over the SIMPLE algorithm to obtain converged solutions more quickly. For the pressure interpolation required when solving the momentum equation, the Body Forced Weighted scheme is used because it is effective for solving the buoyancy natural convection problems. A second-order upwind scheme is chosen for the convection terms in the conservation equations. For turbulent cases, the k- $\varepsilon$ turbulence model with the standard wall function approach is applied. This selection of turbulence model is based on previous experiences and some test calculations. The y+ values closest to the solid walls are within the recommended range for the selected turbulence model. Accordingly, the maximum value of $y+$ closest to the walls is 30 . No particular model for transition between laminar and turbulent flow is adopted as the Rayleigh number is very high for the microgravity case.

The Geometric Reconstruction Scheme is applied for the volume fraction equation to capture the interface.

Three grid systems are used to test the resolution with the total mesh numbers of 12800, 20000 and 30000, respectively. The pressure rises for the two finest grids are almost identical but the results of the coarse grid deviate somewhat. For the finest grid, the numerical solution is too time-consuming for practical use. In this work, accordingly the grid with 20000 quadrilateral grid elements was adopted for the axisymmetric simulations with successively increasing number of meshes towards the walls and interface. While carrying out the computations of transient heat transfer and flow in the fluid domain, the time step of $0.001 \mathrm{~s}$ is chosen to ensure that the Courant number is within 0.1. If the Courant number is larger than 0.1, the time step is reduced appropriately. 
The stream function is formulated as a relation between the streamlines and the statement of conservation of mass. A streamline is a line that is tangent to the velocity vector of the flowing fluid. It is useful in the interpretation of the results. For a $2 \mathrm{D}$ planar flow, the stream function, $\psi$ is defined as

$$
\rho u=\frac{\partial \psi}{\partial y}, \rho v=-\frac{\partial \psi}{\partial x}
$$

where $\psi$ is constant along a streamline and the difference between such values for two neighboring streamlines is proportional to the rate of mass flow between these streamlines. Even this seems not to be correct because this is a 2-D problem and the actual units are $\mathrm{kg} /(\mathrm{m} \cdot \mathrm{s})$, i.e., $\mathrm{kg} / \mathrm{s}$ per unit depth. However, the Fluent software treats 2-D problems as 3-D problems with a unit depth in the $\mathrm{z}$-direction. Thus the units become $\mathrm{kg} / \mathrm{s}$ with this three-dimensional treatment in mind. In this work of $2 \mathrm{D}$ axisymmetric flow, the stream function $\psi$ is defined as:

$$
\rho u_{r}=\frac{1}{r} \frac{\partial \psi}{\partial z}, \rho u_{z}=-\frac{1}{r} \frac{\partial \psi}{\partial r}
$$

where $u_{r}, u_{z}$ are the radial and axial velocity, respectively.

\section{RESULTS AND DISCUSSION}

4.1 Validation case. A validation of the formulation and computational methodology needs to consider the vaporization at the interface. This was done by comparing the predictions with the experimental results by Seo and Jeong [31] for a liquid nitrogen tank. Here a cylindrical tank with a diameter of $0.201 \mathrm{~m}$ and a height of $0.213 \mathrm{~m}$ was investigated. It is filled with liquid nitrogen up to the level of $50 \%$, with a heat leak of $1.2 \mathrm{~W}$. Only nitrogen gas is considered to be present in the ullage space.

The initial pressure was $99000 \mathrm{~Pa}$ and the temperature corresponded to the saturation value at this pressure. The prediction for the pressure variation in the tank is presented in Fig. 2 as the near quasi-equilibrium condition has been reached. The trend of the pressure variation is captured. The pressure rise rate decreases as time progresses and finally approaches a constant value. The numerical pressure is larger than the experimental one in the initial period but the deviation becomes negative at the steady rate value. However, the predictions are observed to be in decent agreement with the experimental data with an average error about $1 \%$ but locally somewhat higher.

4.2 Influence of the gravity value. The effect of gravity on the self-pressurization process was studied for the partially filled tank. For a partially filled tank, the interface between the liquid and vapor is not flat under microgravity conditions as shown in $[32,33]$. Therefore, the steady state distribution in the tank must be determined before the heat flux load is imposed. The fluid is then in equilibrium and treated as a viscous Newtonian fluid with constant properties (including the density) and described by the standard time-dependent continuity and Navier-Stokes equations. Hence, Eqs. (1) and (2) as well as the volume fraction equation can be simplified as given below:

$$
\begin{gathered}
\nabla \cdot \vec{V}=0 \\
\frac{\partial \vec{V}}{\partial t}+(\vec{V} \cdot \nabla) \vec{V}=-\frac{1}{\rho} \nabla p+\vec{g}+\frac{1}{\rho} \nabla \cdot\left[\mu_{e f f}\left(\nabla \vec{V}+\nabla \vec{V}^{T}\right)\right]+\vec{F} \\
\frac{\partial \alpha_{v}}{\partial t}+\vec{V} \nabla \cdot \alpha_{v}=0
\end{gathered}
$$


The no slip and no penetration conditions are used as the boundary conditions at the walls. The distribution of the fluid in the tank at a microgravity of $g=9.81 \times 10^{-3} \mathrm{~m} / \mathrm{s}^{2}=10^{-3} g_{0}\left(g_{0}\right.$ is normal gravity acceleration) and zero-gravity are calculated, as shown in Fig. 3. The contact angle is $\mathbf{5}$ degree as the attempt was to obtain the interface shape before applying heat flux. The liquid climbs up to the top wall along the tank wall due to the surface tension under zero gravity. The interface shape changes until it reaches a steady state from the initial flat interface. In Fig. 3, after the liquid has reached the top wall, it turns to move to the center along the top wall. Liquid accumulates at the center of the top wall like a droplet. The vapor fraction is symmetric but not completely neutral in the center of the tank. No parts of the walls remain dry. A thin liquid film is on the top wall. In microgravity, the surface tension is coupled with the remaining gravity and the coupled effect makes the interface not flat but curved.

A heat flux of $250 \mathrm{~W} / \mathrm{m}^{2}$ is then imposed to investigate the effect of the gravity value under this thermal load.

The heat flux is applied on the side wall of the tank after the steady state configuration has been obtained and the pressure rises for different gravity levels are demonstrated in Fig. 4. The pressure rises more quickly as the gravity level increases during the initial time, as shown in Fig. 4(a). This is so because under microgravity the area of the liquid-wetted wall is larger and it takes more time for the heat to be transferred to the interface without the intensive convection. As time is progressing in Fig. 4(b), the pressure becomes larger and rises faster in zero gravity compared to the case with microgravity. This is so because superheated liquid starts to evaporate and small vapor regions emerge, grow and detach from the tank wall. Figure 5 shows the temperature and streamlines under zero gravity and a reduced gravity as $10^{-3} \mathrm{~g}_{0}$. Rounded isotherms are formed close to the wall while thermal stratification is observed slightly in the center part along the axis. Many separate flow loops are developed near the wall at different heights. This phenomenon can be explained by Fig. 6. The right side of each image is the zoom up of the black box on the left. The heat accumulated at the wall makes the temperature of the liquid higher and higher and evaporation begins to occur as the liquid becomes superheated. Small vapor regions start to be generated on the wall which can be seen in Fig. 6 and the volume of liquid is not equal to unity at the place where the rounded isotherms occur.

As the sidewall heating continues, the vapor region grows and detaches from the tank wall, then moves to the interface under the coupling of the buoyancy force and surface tension or forms a multi-phase liquid-vapor foam. This can be seen in Fig. 7(a) and Fig. 8(a). Fluid vortices are observed close to the vapor regions in Fig. 7(b) and Fig. 8 (b), especially at the place behind the motion of the vapor regions. Usually a clockwise rotation occurs near the tank wall just after the departure of the vapor. The vortices force the vapor to perform a complex motion instead of moving upwards directly. Because of the axial symmetry of the tank and the vortices, the vapor regions from the tank wall merge at the mid axis at different heights with larger vapor regions and move up to the interface. Parts of the vapor arrive at the interface before merging during the flow motion. The velocity around the vortices is larger than the bulk fluid velocity. Isotherms are found around the vapor regions in Fig. 7(c) and Fig. 8(c). As the heat can not be easily carried up to the interface for evaporation, it accumulates at the tank wall. This heat then increases the temperature of tank wall and the liquid propellant. The heat transfer efficiency is low as compared to natural convection. As time elapses, the enthalpy of the heated liquid exceeds the enthalpy according to the tank pressure, and accordingly phase change starts to appear at the tank wall and evaporation becomes the main mode of heat transfer. The heat transfer coefficient increases and more heat is transferred into the tank. Furthermore, if the evaporated liquid adheres at tank wall, hot spot forms may form. These will contribute dangerous to the cryogenic liquid storage.

By comparing Fig. 7 and Fig. 8, it can be seen that the maximum velocity decreases as the gravity acceleration decreases, i.e., $0.2704 \mathrm{~m} / \mathrm{s}$ for $10^{-3} \mathrm{~g}_{0}$ and $0.2677 \mathrm{~m} / \mathrm{s}$ for $0 \mathrm{~g}_{0}$ at the same time. This is because in microgravity the buoyancy force is still important and cannot be ignored. A higher velocity enhances the flow motion and the mixing results in a lower degree of thermal stratification. Therefore, the difference between the maximum and minimum temperatures is larger in zero gravity than in microgravity. 
4.3 Effects of various parameters. The effects of contact angle and surface tension on the selfpressurization were investigated. The simulations were conducted for a microgravity of $10^{-3} \mathrm{~g}_{0}$ with the same heat flux condition on the side wall of the tank. The results of the foregoing analysis are valid for a contact angle of five degrees and a surface tension of $0.002 \mathrm{~N} / \mathrm{m}$. Figure 9 shows the influence of the contact angle while the influence of the surface tension is illustrated in Fig. 10. The pressure and its rise rate decrease as both the contact angle and surface tension increase. This is so because more heat is used for the phase change instead of heating the gas at the interface.

To explain this in more details, an analysis is presented below.

The heat flux partitioning model of Kurul and Podowski has the following structure [34]:

$$
q_{\text {wall }}=q_{c}+q_{q}+q_{e}
$$

The first part is the single-phase heat transfer (convective heat flux):

$$
q_{c}=A_{1} \alpha_{\text {wallscn }}\left(T_{\text {wall }}-T_{l}\right)
$$

$A_{1}$ is the fraction of the wall surface influenced by the liquid. $T_{l}$ is the liquid temperature at the centre of the wall adjacent computational cell and $\alpha_{\text {wallsc }}$ is the wall heat transfer coefficient calculated from the temperature wall function.

The quenching part $q_{q}$ of the wall heat flux $q_{\text {wall }}$ is transported by transient conduction during the time period between the vapor bubble departure and the next vapor bubble formation at the same nucleation site. It is calculated as

$$
q_{q}=A_{2} \alpha_{\text {quench }}\left(T_{\text {wall }}-T_{l}\right)
$$

where $A_{2}$ is the fraction of the wall influenced by vapor bubbles formed at the wall, $\alpha_{\text {quench }}$ is the quenching heat transfer coefficient which has the form:

$$
\alpha_{\text {quench }}=2 f \sqrt{\frac{t_{w} \rho_{l} c_{p l} k_{l}}{\pi}}
$$

The vapor bubble departure frequency $t$ is determined from the following equation:

$$
f=\sqrt{\frac{4 g\left(\rho_{l}-\rho_{v}\right)}{3 d_{b w} \rho_{l}}}
$$

The waiting time $t_{w}$ is the difference between the vapor bubble time period and the growth time $t_{g}$ :

$$
t_{w}=1 / f-t_{g}
$$

The heat flux of vaporization $q_{e}$ can be computed by the following equation:

$$
q_{e}=\frac{\pi}{6} d_{b w}^{3} \rho_{v} n f L_{H}
$$

where $d_{b w}, n$ and $t$ are the departure diameter of a vapor bubble, the number of active nucleation sites and vapor bubble departure frequency, respectively. The departure diameter is calculated by the Kirichenko et al. equation [35]:

$$
d_{b w}=C_{b w}\left(\frac{3}{2} \frac{\sigma}{g\left(\rho_{l}-\rho_{v}\right)} \frac{2 \sigma T_{\text {sat }}}{L_{H} \rho_{v} \Delta T_{\text {sup }}}\right)^{1 / 3}
$$


The active nucleation site density $n$ is correlated to the wall superheat as:

$$
n=C_{n}\left(\frac{L_{H} \rho_{v} \Delta T_{\text {sup }}}{\sigma T_{\text {sat }}}\right)^{m}
$$

In eq. (30), $C_{n}$ and $m$ are determined by:

$$
\left\{\begin{array}{l}
P / P_{c r}>0.04 \sim 0.06: C_{n}=10^{-7}, m=2 \\
P / P_{c r}<0.02 \sim 0.04: C_{n}=6.25 \times 10^{-14}, m=3
\end{array}\right.
$$

The evaporation part $q_{e}$ contributes to the pressure rise. The other two parts of the wall heat flux are mainly increasing the temperature of the whole system. From the above description, the contact angle and surface tension affect the evaporation heat flux through the vapor bubble dynamics. Zeng et al. [36] introduced a lattice Boltzmann method to simulate the bubble growth in pool boiling and concluded that the influence of the contact angle on the departure diameter of a vapor bubble can be neglected. However, the departure frequency decreases as the contact angle increases. Thus the heat flux of vaporization decreases according to Eq. (28) and this results in a decrease of the pressure magnitude and its rise rate.

By combining Eqs. (26) - (31), the relation between the evaporation heat flux and the surface tension is obtained as:

$$
q_{e} \propto \sigma^{2-\left(m+\frac{1}{3}\right)}
$$

If $m=2$ or $m=3$, the exponent is negative. Therefore, the heat flux $q_{e}$ decreases as the surface tension $\sigma$ increases as shown in Fig. 10. Less heat is transferred to the vapor part through vaporization which contributes to the pressure rise at the same wall heat flux.

Vortices are also observed at the place just behind the vapor bubble. The direction of the loops is reversed compared to the bulk fluid circulation. The warm liquid heated by the wall moves no longer just up along the wall to the interface for evaporation and falls down again in the middle in the presence of bubbles. The motion is much more complicated.

\section{CONCLUSIONS}

Simulations of self-pressurization in a partially filled liquid hydrogen tank were conducted with the CLSVOF method, mass and heat transfer models to understand the effect of evaporation on self-pressurization. The capabilities of the models were validated by existing experimental data. Small vapor regions emerge when the heat transfer mode turns from convection to phase change by evaporation and this changes the pressure rise. The pressure is larger in microgravity of $10^{-3} \mathrm{~g}_{0}$ compared to the zero gravity condition in the initial period. After evaporation has emerged, the situation becomes inversed. The dynamics of the vapor regions induce vortices in the fluid and the bulk fluid is not just a simple large circulation region with one direction. The vapor regions move to the center after detaching from the tank wall and towards the interface as a large vapor region. The velocity around the vortices is larger than that of the bulk fluid. The pressure is decreased by an increase of the contact angle and surface tension because such increases change the dynamics of the vapor phase regions as the theoretical vapor bubble departure diameter is affected. The direction of the vortices behind the vapor region is observed to be reversed compared to the bulk fluid circulation. The bubble dynamics and its influence on the heat transfer need further investigation.

\section{ACKNOWLEDGMENTS}

The work was supported in part by the Natural Science Foundation of China under Grant No. 91216201 and 51205403. Additional resources were provided by the Department of Energy Sciences at Lund University. Also the Swedish National Infrastructure for Computing (SNIC) provided efficient computing resources. 
These supports are kindly acknowledged. The support provided by China Scholarship Council (CSC) during the visit of Juan Fu to Lund University is also kindly acknowledged.

\section{REFERENCES}

[1] S. Barsi, M. Kassemi, Investigation of tank pressurization and pressure control - Part I: Experimental Study, ASME J. Therm. Sci. Eng. Appl. 5 (2013) p. no. 041005.

[2] S. Barsi, M. Kassemi, Investigation of tank pressurization and pressure control - Part II: Numerical Modeling, ASME J. Therm. Sci. Eng. Appl. 5 (2013) p. no. 041006.

[3] J. Aydelott, Normal gravity self-pressurization of 9-inch $(23 \mathrm{~cm})$ diameter spherical liquid hydrogen tanks, NASA TND-4171 (1967) USA.

[4] J.I. Hochstein, H.C. Ji, J. Aydelott, Prediction of self-pressurization rate of cryogenic propellant tankage, J. Propulsion and Power, 6(1) (1990) 11-17.

[5] K. Abdalla, T. Frysinger, C. Androcchio, Pressure-rise characteristics for a liquid hydrogen dewar for homogeneous, normal gravity, quiescent, and zero-gravity tests, NASA TM X-1134, (1965) USA.

[6] N. Amirkhanyan, S. Cherkasov, Theoretical analysis and procedure for the calculation of thermophysical processes occurring in a cryogenic vessel under conditoins of nonvented storage, J. High Temperature, 39 (2001) 905-911.

[7] S. Baris, M. Kassemi, Numerical and experimental comparisons of the self-pressurization behavior of an LH2 tank in normal gravity, J. Cryogenics 48 (2008) 122-129.

[8] C. Panzarella, M. Kassemi, On the validity of purely thermodynamic descriptions of two phase cryogenic fluid storage, J. Fluid Mechanics 484 (2003) 41-68.

[9] N.V. Dresar, C. Lin, M. Hasan, Self-pressurization of a flightweight liquid hydrogen tank: effects of fill level at low wall heat flux, NASA TM 105411 (1992) USA.

[10] S. Roh, G. Son, Numerical study of natural convection in a liquefied natural gas tank, J. Mechanical Science and Technology 26 (2012) 3313-3140.

[11] G. Grayson, A. Lopez, F. Chandler, L. Hastings, S. Tucker, Cryogenic tank modeling for the saturn AS203 experiment, AIAA Paper no. 2006-5258 (2006).

[12] J. Fu, B. Sunden, X.Q. Chen, Analysis of self-pressurization phenomenon in a cryogenic fluid storage tank with VOF method, Proceedings of the ASME 2013 International Mechanical Engineering Congress \& Exposition, IMECE2013-63209 (2013).

[13] J. Fu, B. Sunden, X.Q. Chen, Influence of wall ribs on the thermal stratification and self-pressurization in a cryogenic liquid tank, Applied Thermal Engineering 73 (2014) 1421-1431.

[14] A. Albadawi, D.B. Donoghue, A.J. Robinson, D.B. Murray, Y.M.C. Delauré, Influence of surface tension implementation in Volume of Fluid and coupled Volume of Fluid with Level Set methods for bubble growth and detachment, Int. J. Multiphase Flow 53 (2013) 11-28.

[15] C.W Hirt, B.D. Nichols, Volume of fluid (VOF) method for the dynamics of free boundaries, J. Computational Physics 39 (1981) 201-225.

[16] A.J. Luckmann, M.V.C. Alves, J.R. Barbosa Jr., Analysis of oil pumping in a reciprocating compressor, Applied Thermal Engineering 29 (2009) 3118-3123.

[17] G. Li, S. Frankel, J.E. Braun, E.A. Groll, Application of CFD model to two-phase flow in refrigeration distributors, J. HVAC \& R Research 11 (2005) 45-62.

[18] M. Sussman, K.M. Smith, M.Y. Hussaini, M. Ohta, R. Zhi-Wei, J. Computational Physics 221 (2007) 469-505.

[19] J.U. Brackbill, D.B. Kothe, C. Zemach, A continuum method for modeling surface tension, J. Computational Physics, 100, 335-354, 1992.

[20] K. Xu, B. Ruan, H. Meng, A thermal performance factor for evaluation of active engine cooling with asymmetric heating, Applied Thermal Engineering 73 (2014) 351-356.

[21] J. Wang, J. Wang, Y. Dai, P. Zhao, Thermodynamic analysis and optimization of a transcritical $\mathrm{CO}_{2}$ geothermal power generation system based on the cold energy utilization of LNG, Applied Thermal Engineering 70 (2014) 531-540.

[22] L. Wang, Z. Chen, H. Meng, Numerical study of conjugate heat transfer of cryogenic methane in rectangular engine cooling channels at supercritical pressures, Applied Thermal Engineering 54 (2013) 237-246. 
[23] D. Sun, J. Xu, Q. Chen, Modeling of the evaporation and condensation phase-change problems woith FLUENT, Numerical Heat Transfer, Part B, 66 (2014) 326-342.

[24]D. Sun, J. Xu, L. Wang, Development of a vapor-liquid phase change model for volume-of-fluid method in FLUENT, International Communications Heat and Mass Transfer 39 (2012) 1101-1108.

[25] Z. Pan, J.A. Weibel, S.V. Garimella, Spurious current suppression in VOF-CSF simulation of slug flow through small channels, Numerical Heat Transfer, Part A, 67 (2015) 1-12.

[26] H. Lee, A Pressure Iteration scheme for two-phase flow modeling, T.N. Veziroglu (Ed.), Multiphase Transport Fundamentals, Reactor Safety, Applications, 1, Hemisphere Publishing, Washington, DC, (1980) .

[27] Z.Y. Liu, B. Sunden, J.L. Yuan, VOF modeling and analysis of filmwise condensation between vertical parallel plates, Heat Transfer Research, 43 (2012) 47-68,.

[28] S. Osher, J. A. Sethian, Fronts propagating with curvature-dependent speed: Algorithms based on Hamilton-Jacobi formulations, J. Computational Physics 79 (1988) 12-49.

[29] S.P. Kumar, B.V.S.S.S Prasad, G. Venkatarathnam, K. Ramamurthi, S.S. Murthy, Influence of surface evaporation on stratification in liquid hydrogen tanks of different aspect ratios, Int. J. Hydrogen Energy, 32 (2007) 1954-1960.

[30] S. Gursu, S. A. Sherif, T. N. Veziroglu, J.W. Sheffield, Analysis and optimization of thermal stratification and self-pressurization effects in liquid hydrogen storage systems, Part 1: Model development, ASME J. Energy Resources Technology, 115 (1993) 221-227.

[31] M. Seo, S. Jeong, Analysis of self-pressurization phenomenon of cryogenic fluid storage tank with thermal diffusion model, J. Cryogenics, 50 (2010) 549-555.

[32] J.A. Stark, R. D. Bradshow, M.H. Blatt, Low-gravity fluid behavior technology summaries, NASA-CR134748. 1974 USA.

[33] S. Ostrach, Low-gravity fluid flows, Ann. Rev. Fluid Mechanics 14 (1974) 313-345.

[34] N. Kurul, M.Z. Podowski, Multidimensional effects in forced convection subcooled boiling, Proc. 9th Int. Heat Transfer Conference 1 (1990) 21-26.

[35] Y. A Kirichenko, L A Slobozhanin, N. S. Shcherbakova, Analysis of quasi-static conditions of boiling onset and bubble departure, J. Cryogenics 23 (1983) 110-112.

[36] J.B. Zeng, L.J. Li, Q. L, F.M. J, Simulation of bubble growth process in pool boiling using lattice Boltzmann method, Acta Phys. Sin. 60 (2011) 066401. 


\section{Figure Captions}

Fig. 1 Physical model of the partially filled cylindrical tank.

Fig. 2 Comparison of the computed pressure variations with the experimental data of [21].

Fig. 3 Steady state distributions of the liquid and vapor in a tank at different gravities (a) $0 \mathrm{~g}_{0}$ and (b) $10^{-3} \mathrm{~g}_{0}$

Fig. 4 Self-pressurization in a partially filled liquid hydrogen tank at different gravities.

Fig. 5 Isocontours of temperature and streamfunction of self-pressurization for two gravities.

Fig. 6 Volume of liquid at different gravities.

Fig. 7 (a) The volume of fluid, (b) vector of velocity, (c) temperature of self-pressurization for $0 \mathrm{~g}_{0}$ at $170 \mathrm{~s}$.

Fig. 8 (a) The volume of fluid, (b) vector of velocity, (c) temperature of self-pressurization for $10^{-3} \mathrm{~g}_{0}$ at $170 \mathrm{~s}$

Fig. 9 (a) Self-pressurization in a partially filled liquid hydrogen tank for different contact angles, (b) volume of liquid and stream function of self-pressurization for different contact angles at 170s

Fig. 10 (a) Self-pressurization in a partially filled liquid hydrogen tank for different values of the surface tension, (b) volume of liquid and stream function of self-pressurization for different values of the surface tension at $170 \mathrm{~s}$ 


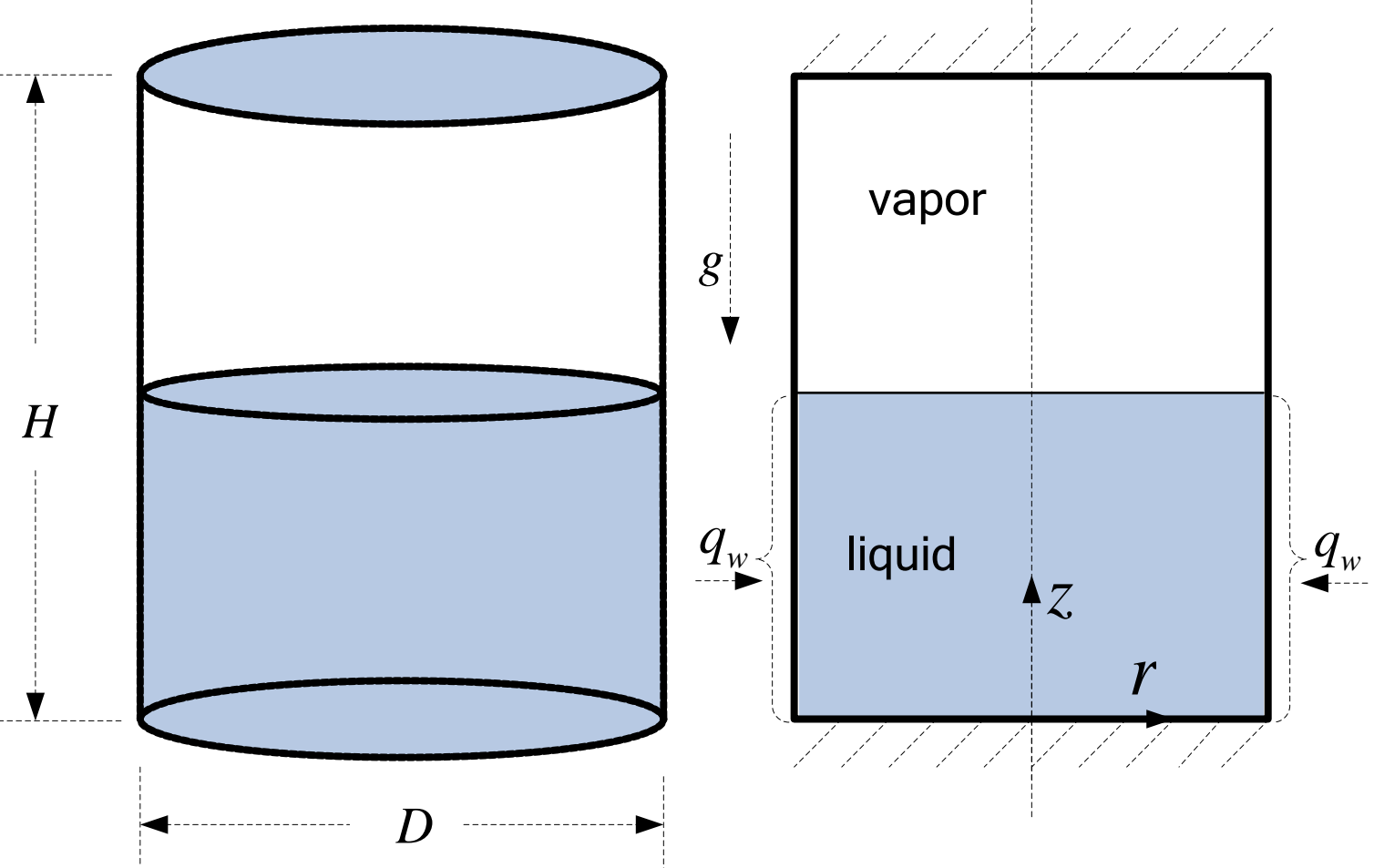

Figure 1 Physical model of the partially filled cylindrical tank. 


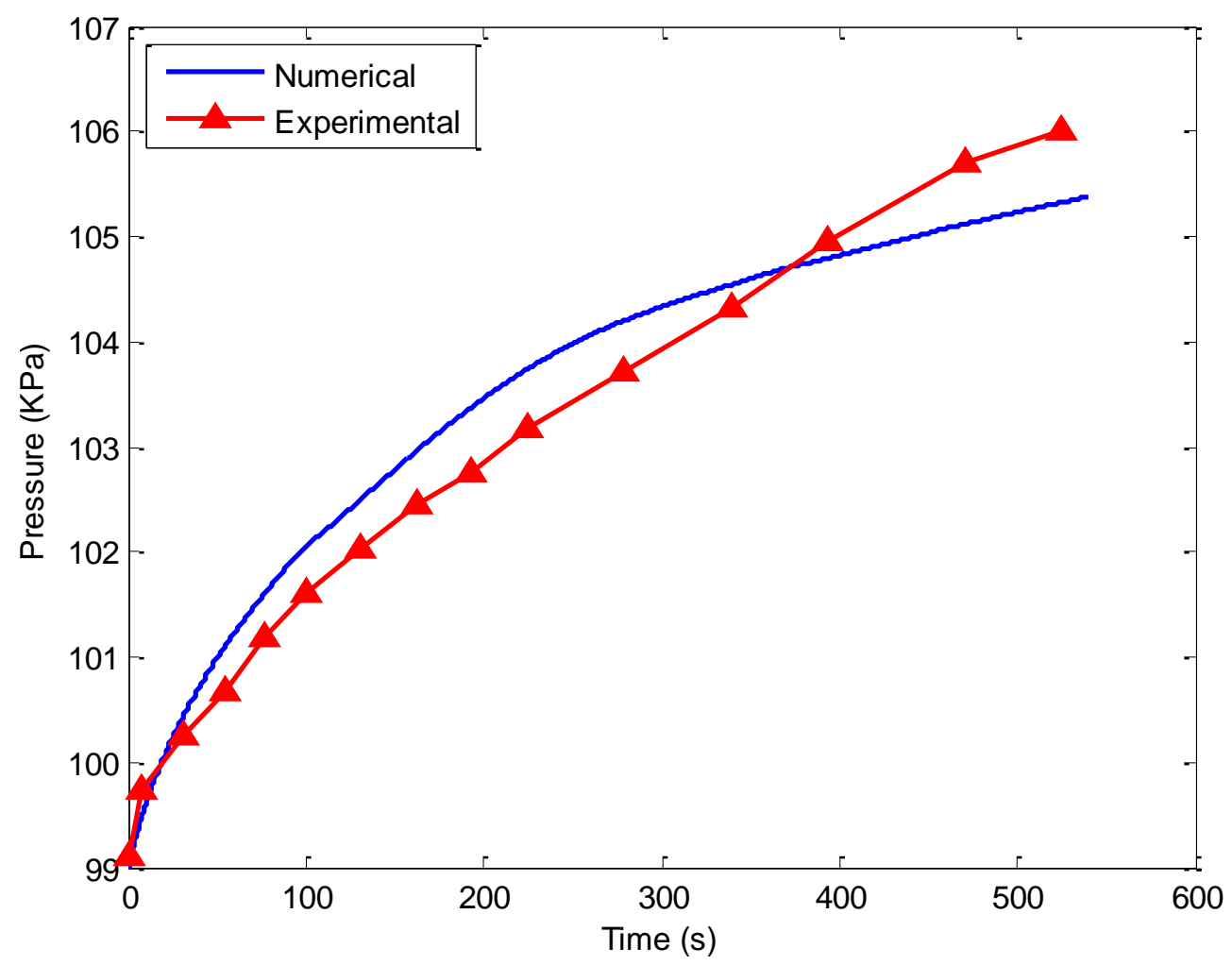

Figure 2 Comparison of the computed pressure variations with the experimental data of [21]. 


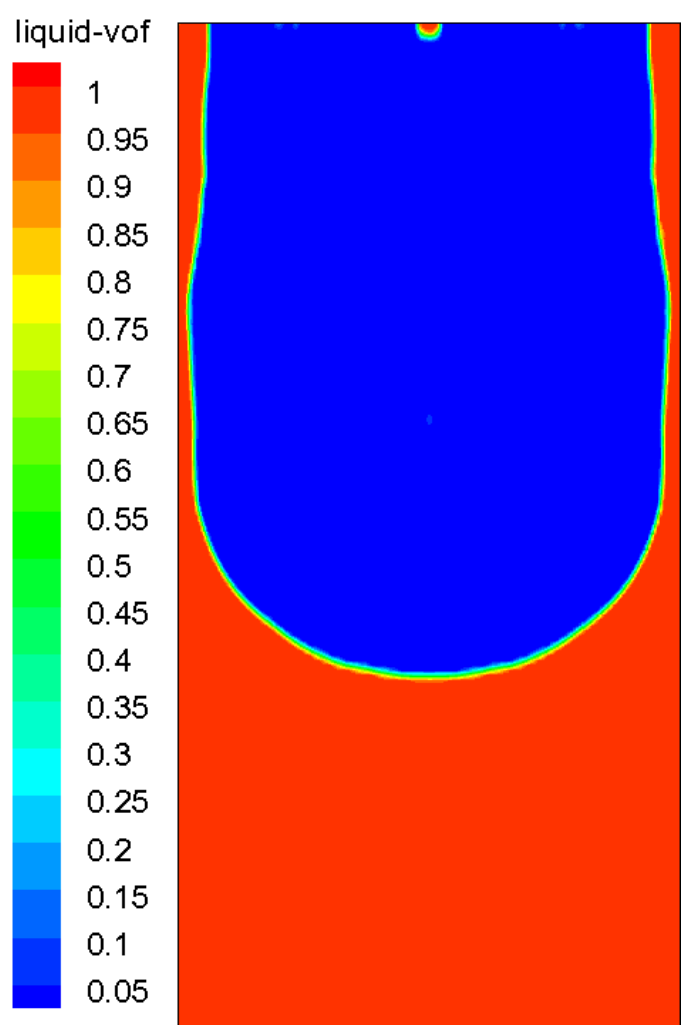

(a) $0 \mathrm{~g}_{0}$

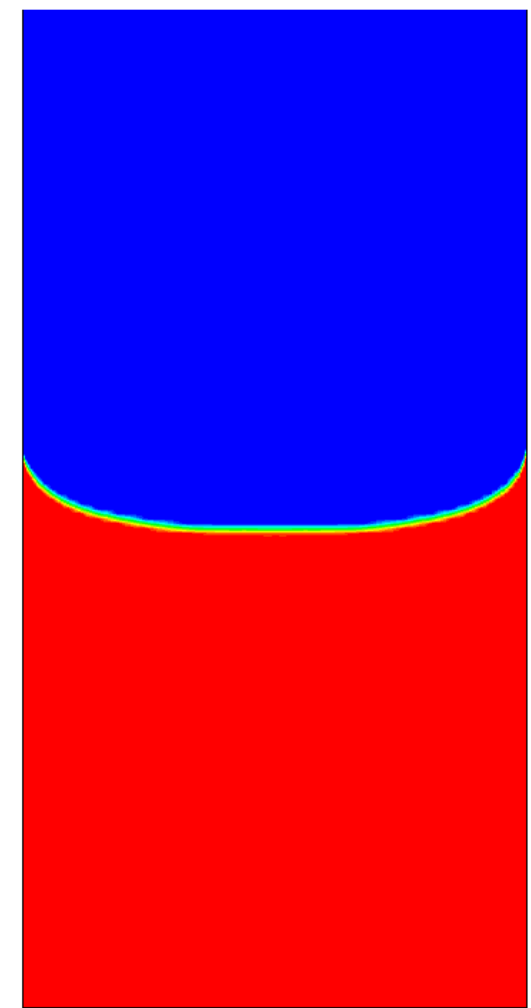

(b) $10^{-3} \mathrm{~g}_{0}$

Figure 3 Steady state distributions of the liquid and vapor in a tank at different values of the gravity 


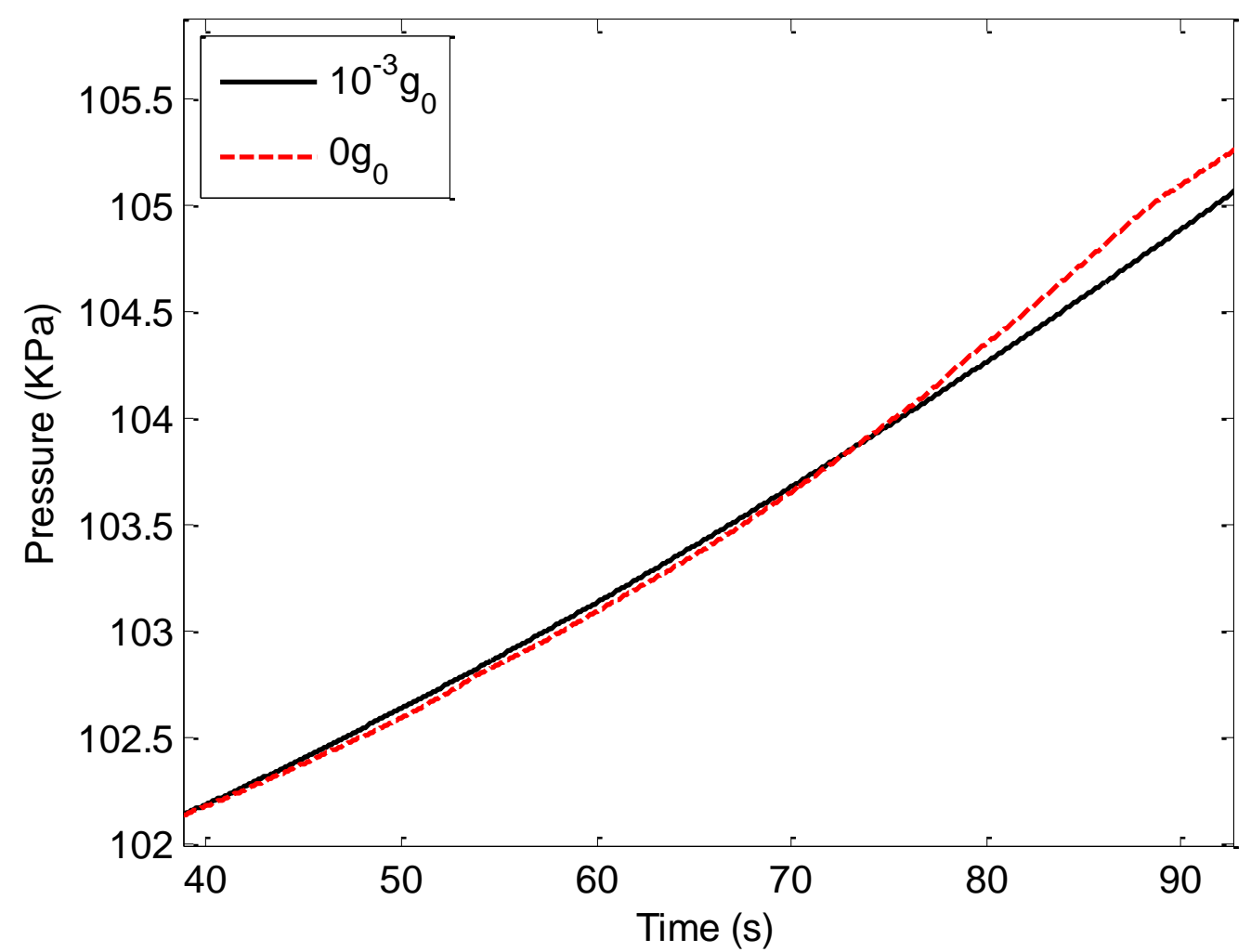

(a) Initial time

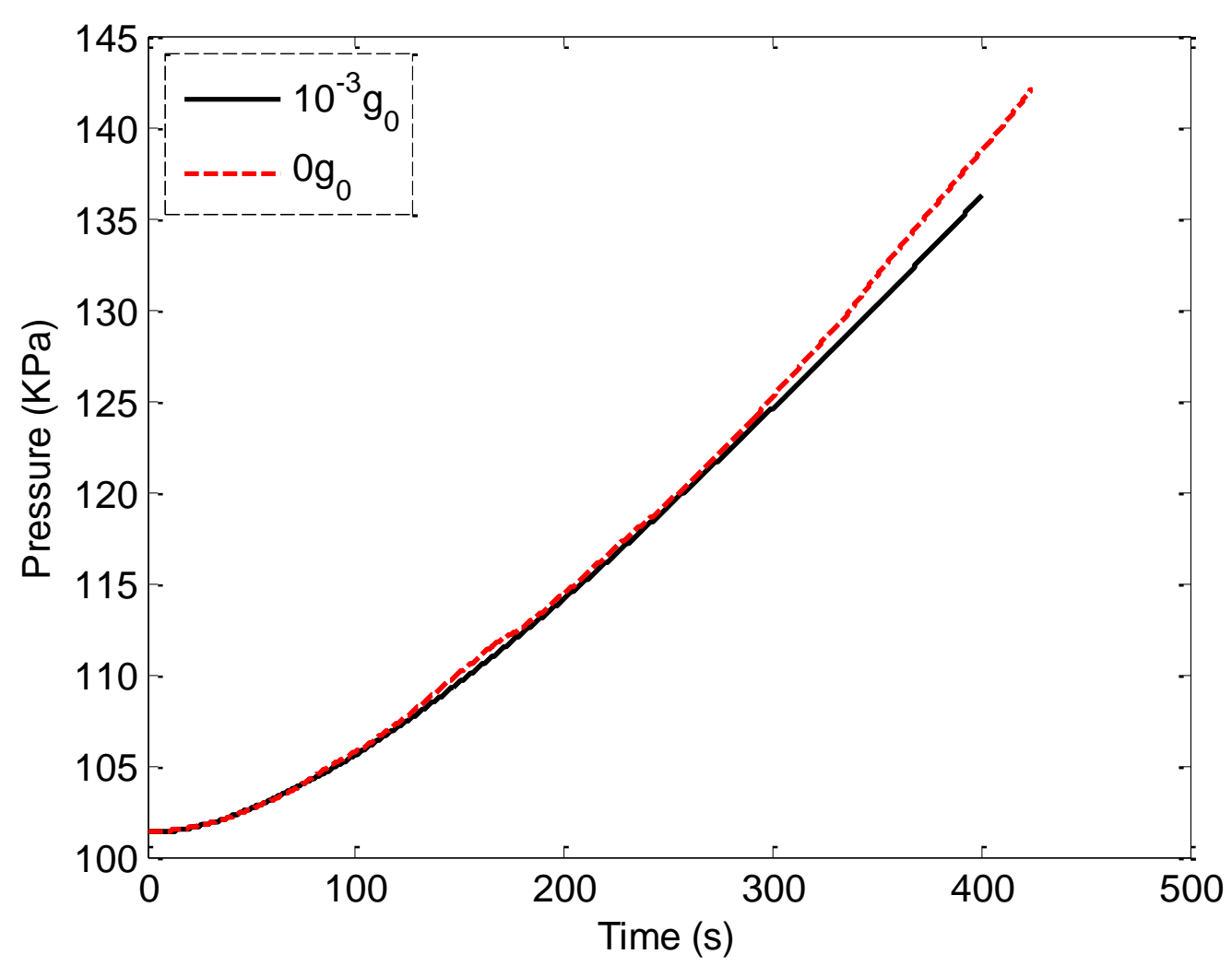

(b) Relatively long time

Figure 4 Self-pressurization in a partially filled liquid hydrogen tank at different gravities. 


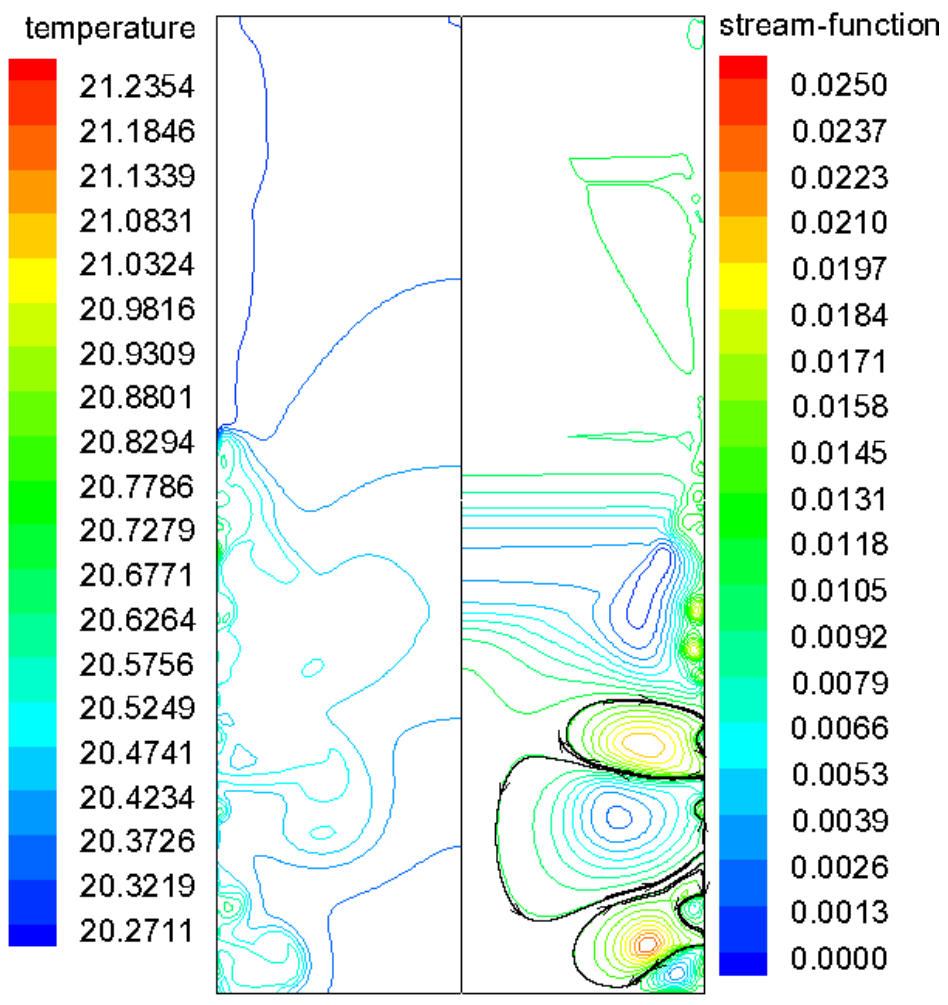

(a) $0 \mathrm{~g}_{0}$

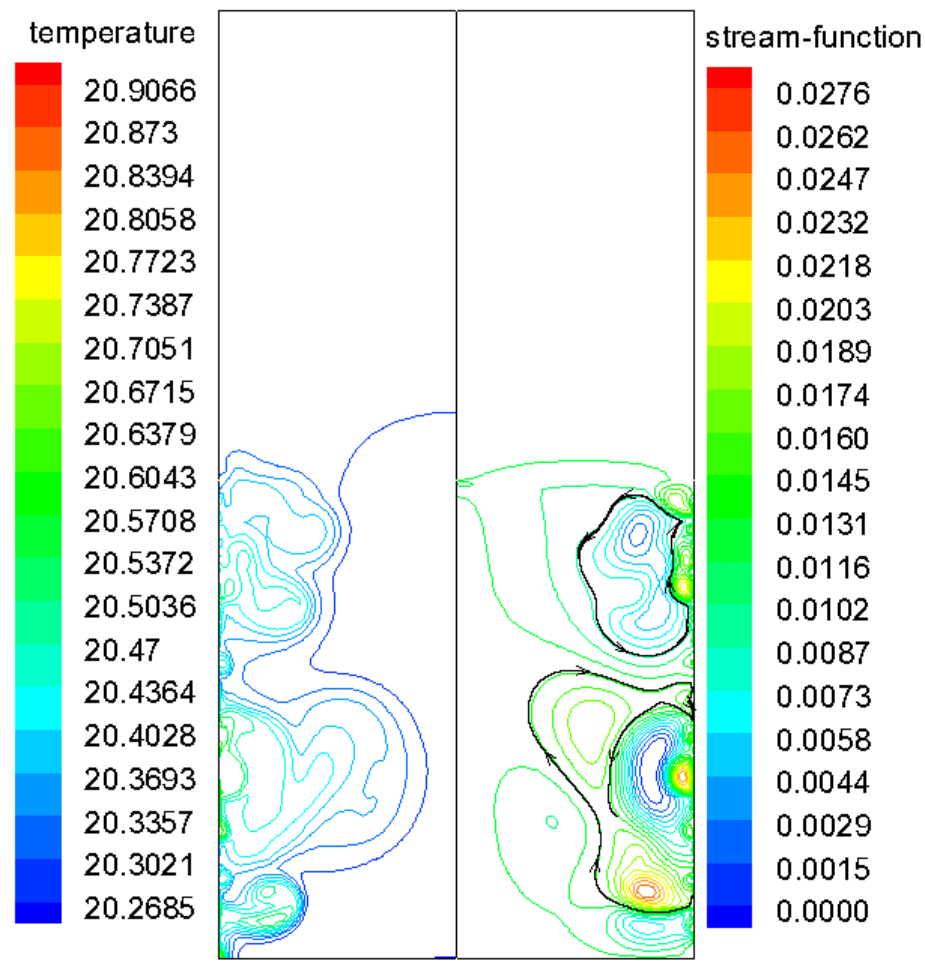

(b) $10^{-3} \mathrm{~g}_{0}$

Figure 5 Isocontours of temperature and streamfunction of self-pressurization for two gravity values. a) zero gravity, b) microgravity 


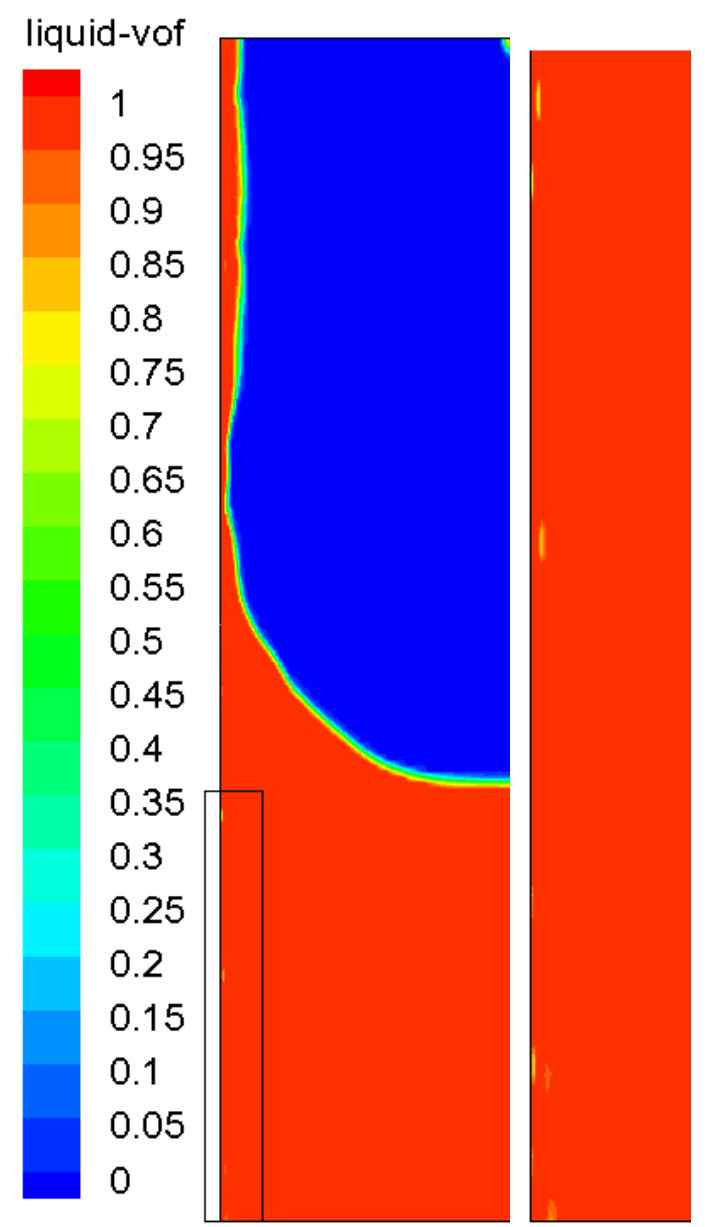

(a) $0 \mathrm{~g}_{0}$

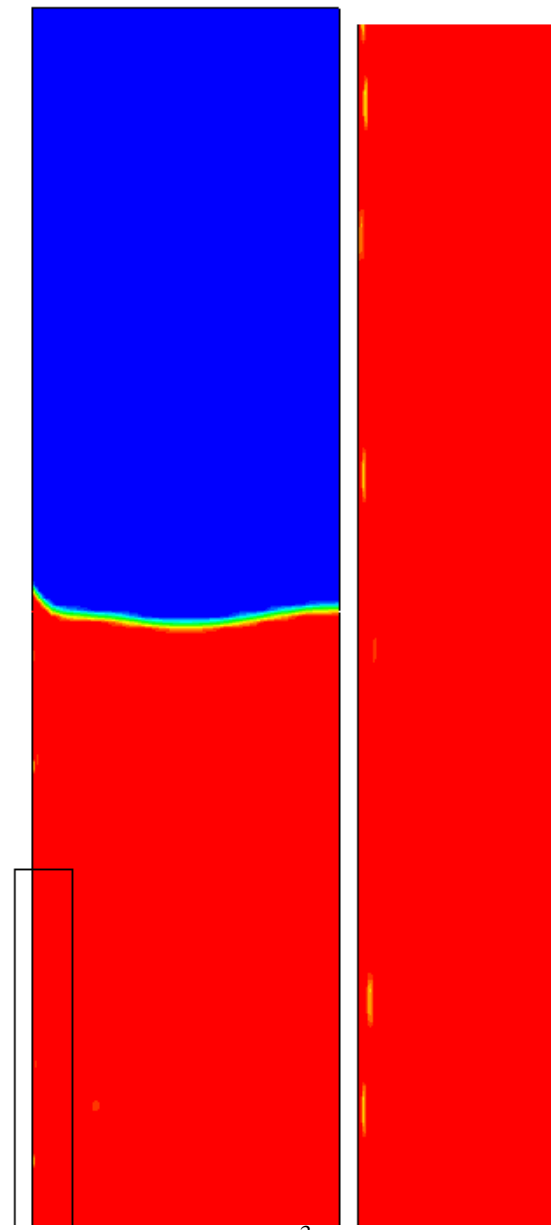

(b) $10^{-3} \mathrm{~g}_{0}$

Figure 6 Volume of liquid at different gravities. 


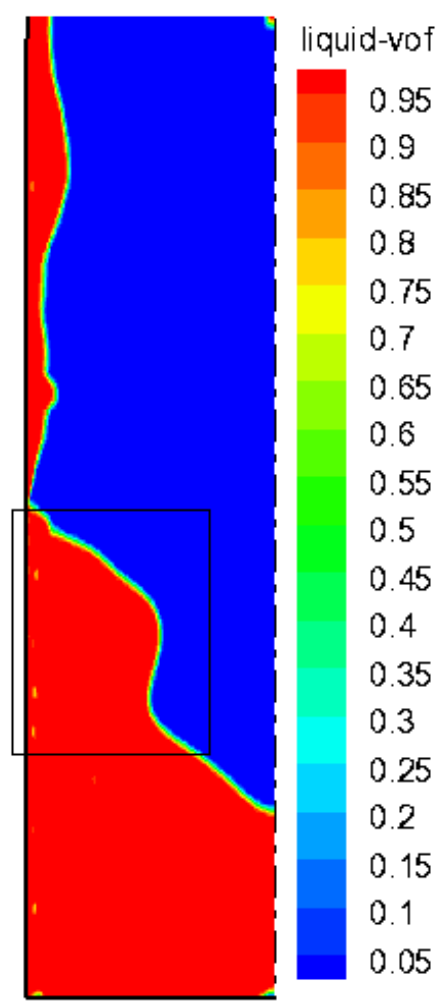

(a)

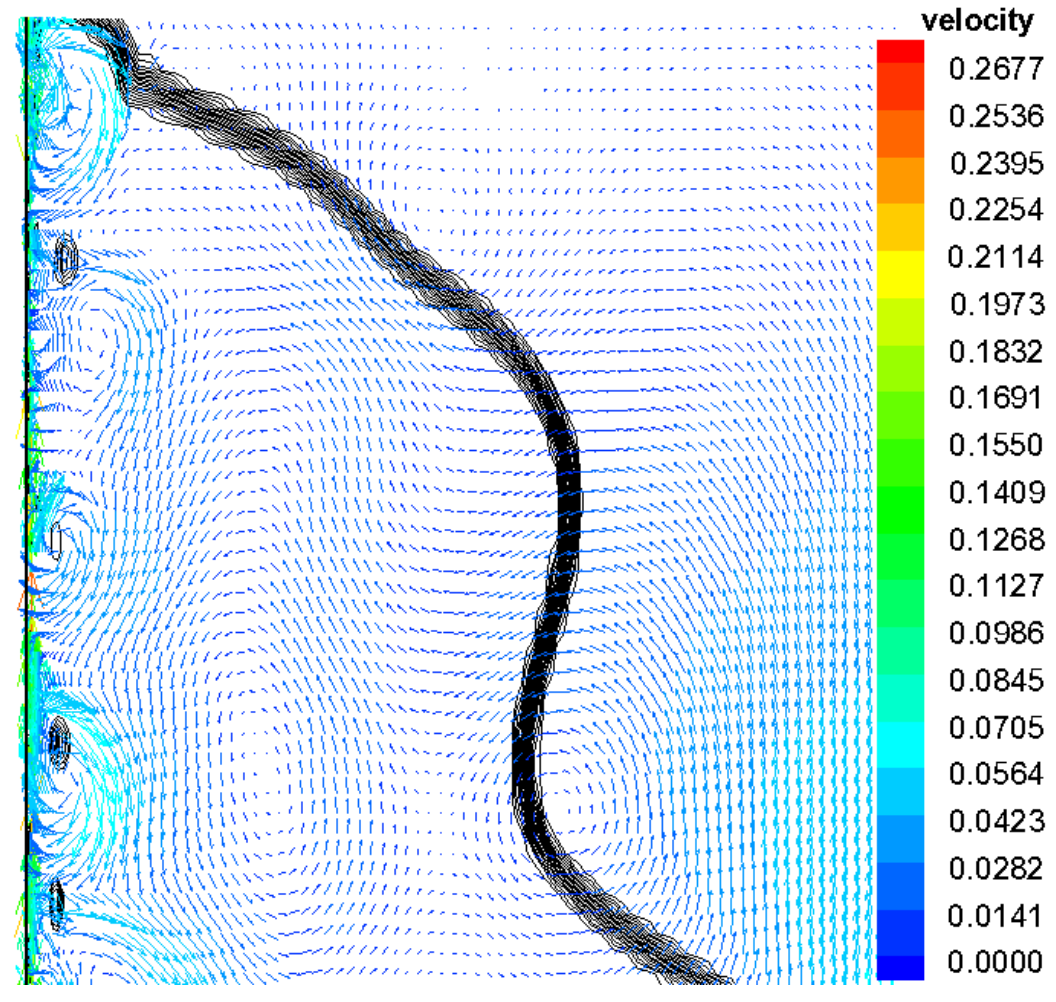

(b)

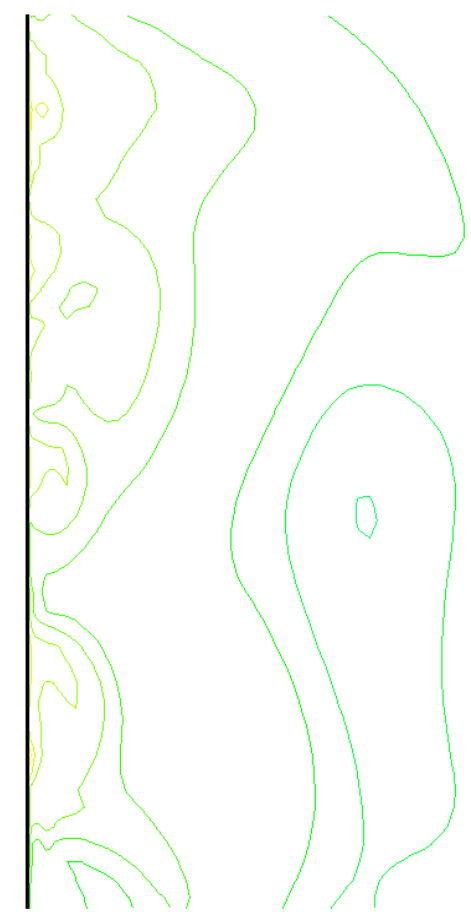

\begin{tabular}{|l|}
\hline temperature \\
\hline 21.1152 \\
21.0714 \\
21.0276 \\
20.9838 \\
20.9399 \\
20.8961 \\
20.8523 \\
20.8085 \\
20.7647 \\
20.7209 \\
20.677 \\
20.6332 \\
20.5894 \\
20.5456 \\
20.5018 \\
20.458 \\
20.4141 \\
20.3703 \\
20.3265 \\
20.2827
\end{tabular}

(c)

Figure 7 (a) The volume of fluid, (b) vector of velocity $(\mathrm{m} / \mathrm{s})$, (c) temperature (K) of self-pressurization for $0 \mathrm{~g}_{0}$ at $170 \mathrm{~s}$. 


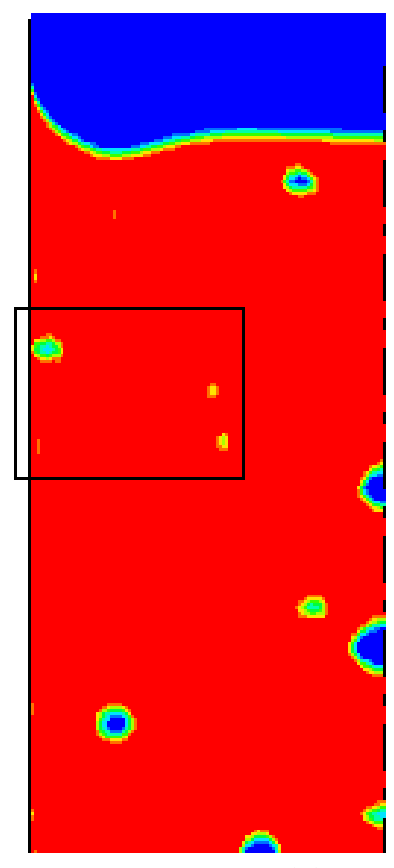

(a)
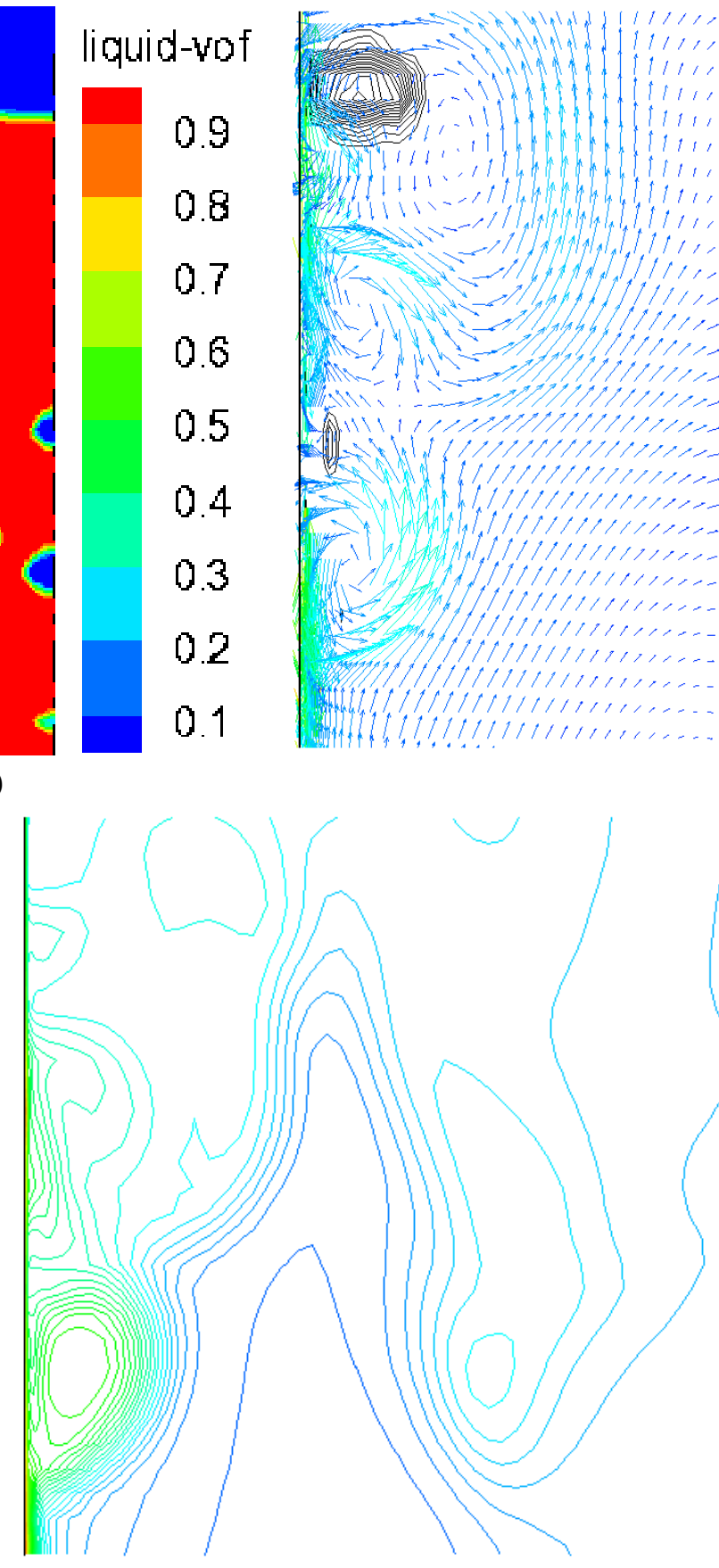

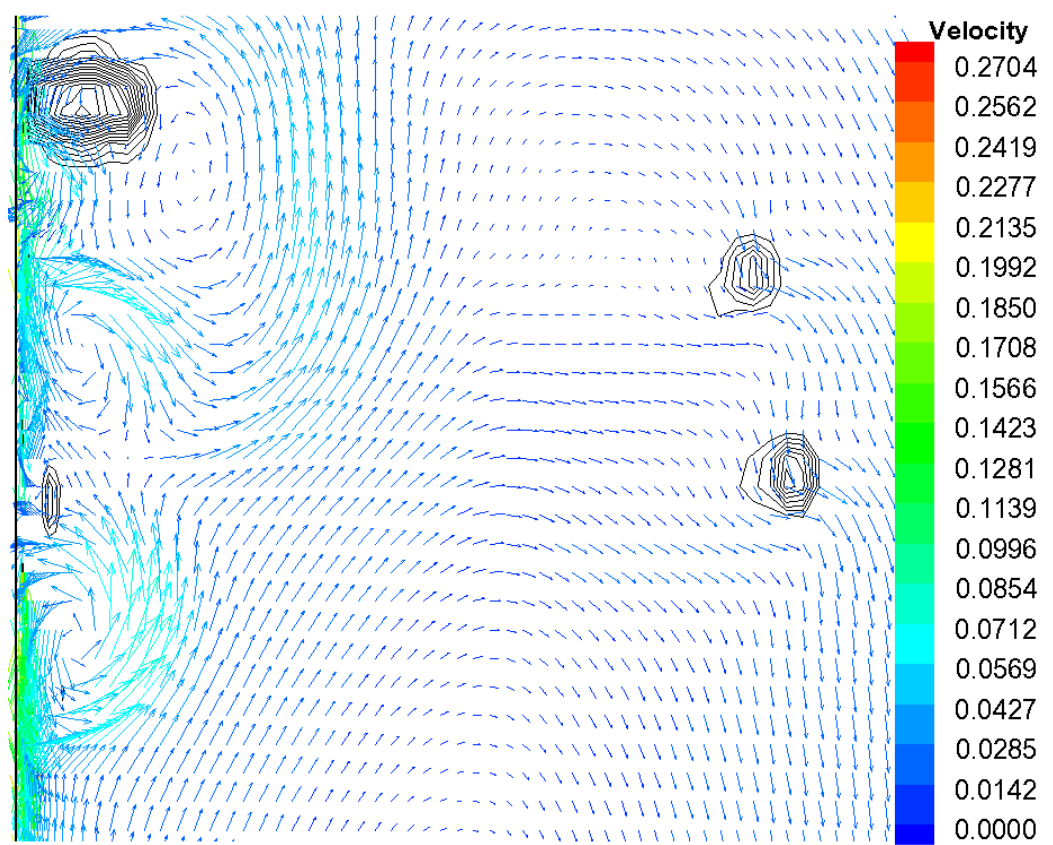

(b)

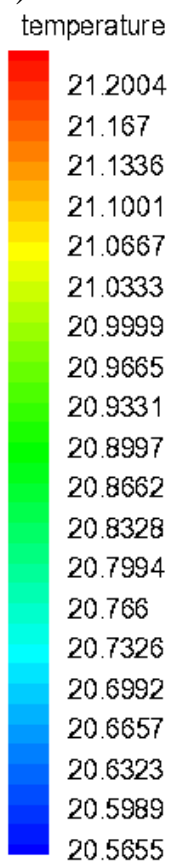

(c)

Figure 8 (a) The volume of fluid, (b) vector of velocity $(\mathrm{m} / \mathrm{s})$, (c) temperature $(\mathrm{K})$ of self-pressurization for $10^{-3} \mathrm{~g}_{0}$ at $170 \mathrm{~s}$ 


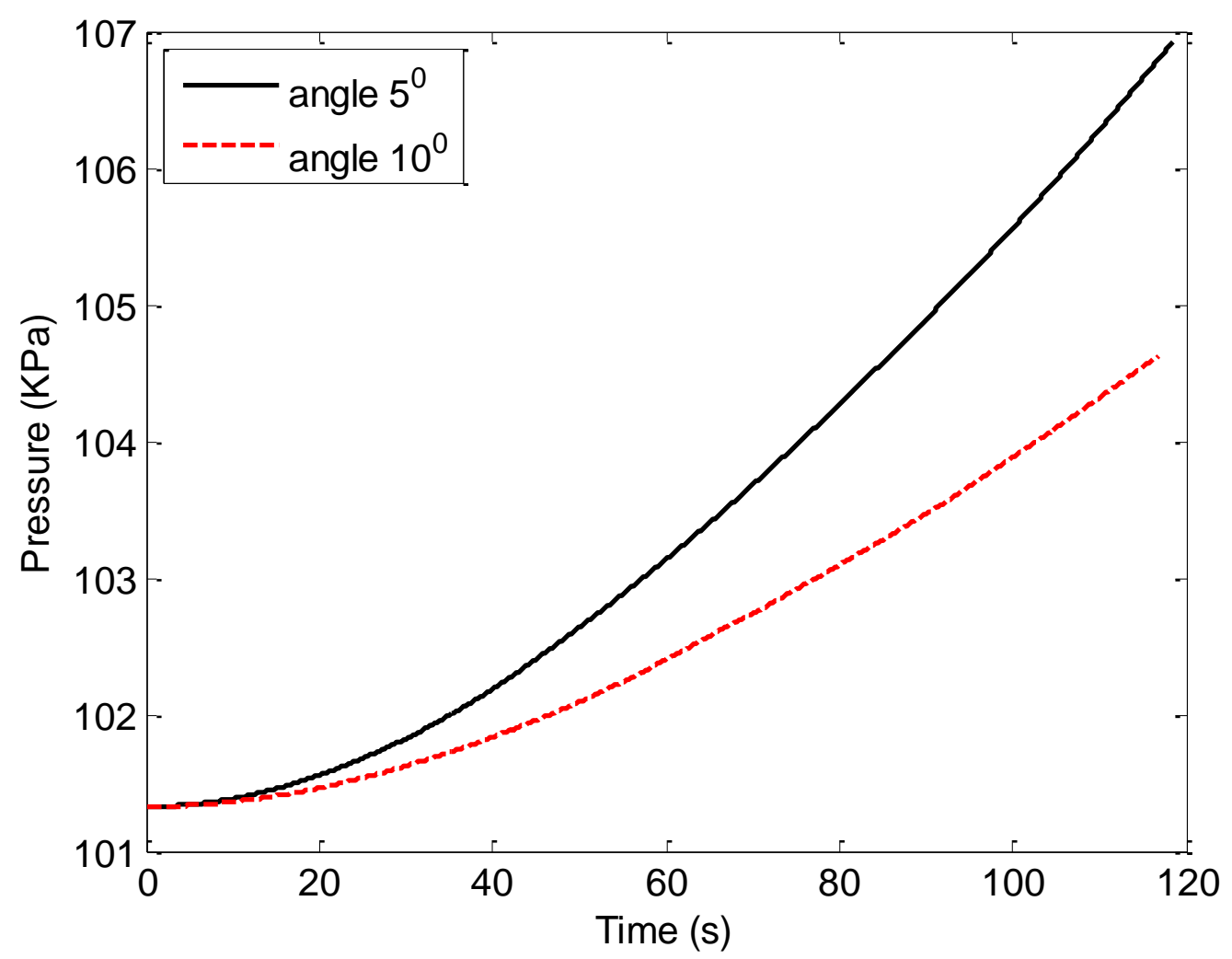

(a)

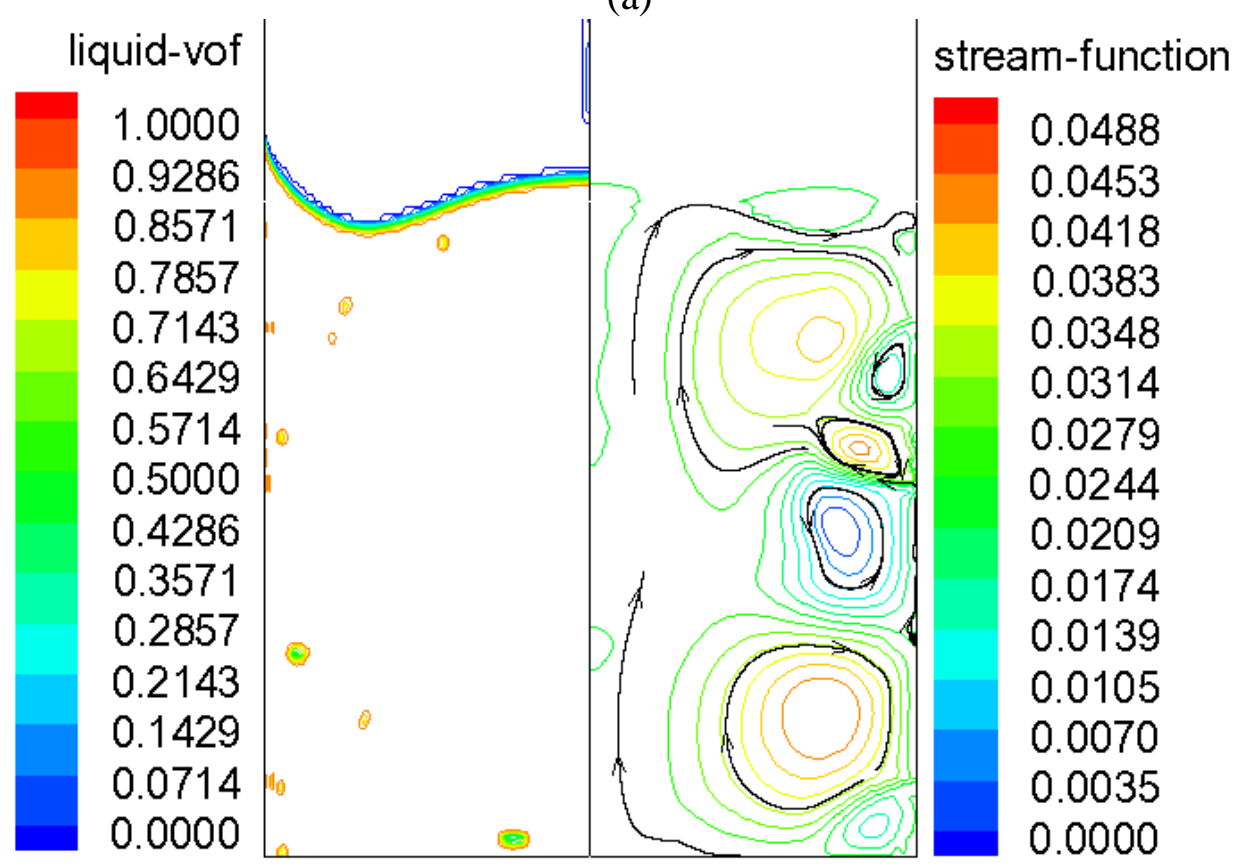

(b)

Figure 9 (a) Self-pressurization in a partially filled liquid hydrogen tank for different contact angles, (b) volume of liquid and stream function of self-pressurization for contact angle of 10 degree at 170s 


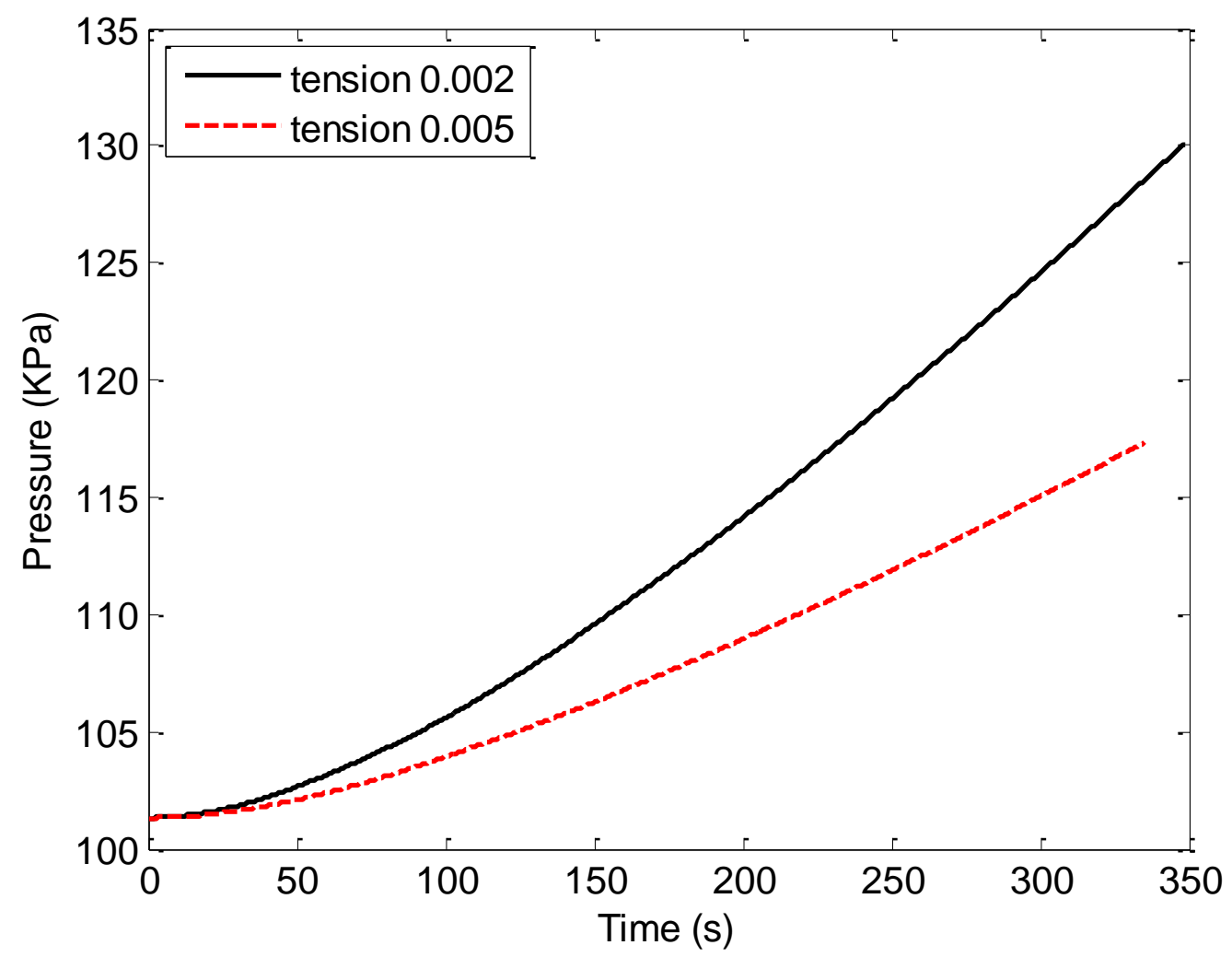

(a)

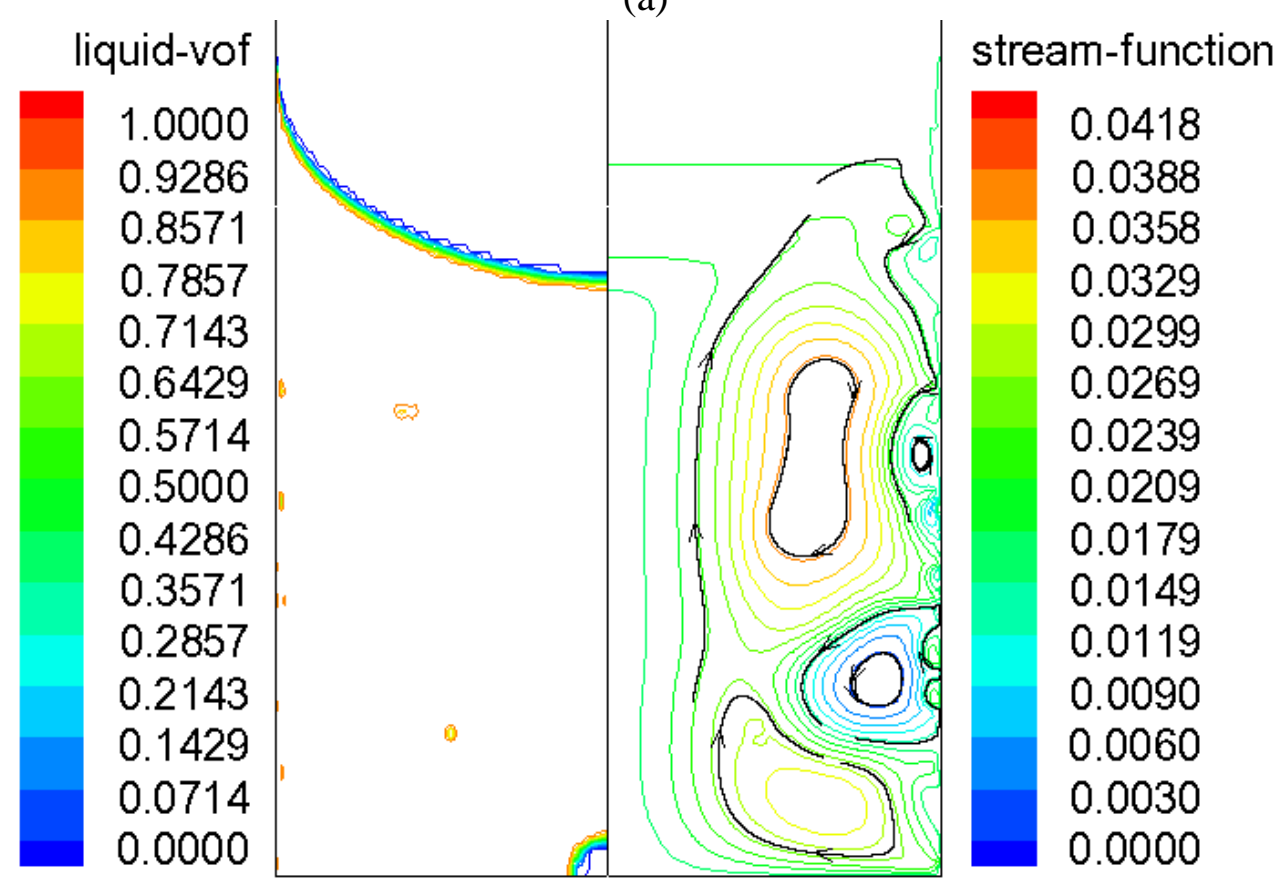

(b)

Figure 10 (a) Self-pressurization in a partially filled liquid hydrogen tank for different values of the surface tension, (b) volume of liquid and stream function of self-pressurization for the surface tension of $0.005 \mathrm{~N} / \mathrm{m}$ at $170 \mathrm{~s}$ 